\title{
PSA Screening for Prostate Cancer in Men under the Age of 65: A Review of Current Practice
}

\author{
Andrew H. Khalifa1, Joel Z. Cornfield ${ }^{2}$ \\ ${ }^{1}$ St. James School of Medicine, Anguilla, The Grenadines \\ ${ }^{2}$ Attending Staff Adventist Hinsdale Hospital and Jesse Brown Veteran's Administration Hospital, Chicago, USA \\ Email: jcornfield@uropartners.com, joel.cornfield@va.gov
}

How to cite this paper: Khalifa, A.H. and Cornfield, J.Z. (2018) PSA Screening for Prostate Cancer in Men under the Age of 65: A Review of Current Practice. Open Journal of Urology, 8, 135-160. https://doi.org/10.4236/oju.2018.85015

Received: April 2, 2018

Accepted: May 8, 2018

Published: May 11, 2018

Copyright $(9) 2018$ by authors and Scientific Research Publishing Inc. This work is licensed under the Creative Commons Attribution International License (CC BY 4.0).

http://creativecommons.org/licenses/by/4.0/

\section{(c) (i) Open Access}

\begin{abstract}
Purpose: To review current practice in the use of Prostate Specific Antigen (PSA) testing and assess the overall risks and benefits of PSA testing for Adenocarcinoma of the Prostate, specifically in men under age 65, and those living with risk factors for the disease. Materials and Methods: An extensive literature review of PSA testing in various age groups was conducted with the goal being to review PSA screening trends and assess current recommendations regarding PSA Screening for Adenocarcinoma of the Prostate in various age groups, focusing on studies directed towards patients under the age of 65 and the long-term risks and benefits of PSA screening in the diagnostic algorithm. In order to reduce bias, we assessed our sources for variables such as reporting, measurement and/or selection bias. Our conclusions were then compared with current recommendations. Relevant studies with data compiled after 2004, centered around PSA screening in men under the age of 65 in general and specifically in men with risk factors for developing Adenocarcinoma of the Prostate were included. Editorials, newspaper articles and other non-scholarly and non-peer reviewed sources were automatically excluded.
\end{abstract}

\section{Keywords}

Adenocarcinoma, Adenocarcinoma of the Prostate, Aggressive Disease

\section{Introduction}

Prostate Specific Antigen (PSA) is a serine protease glycoprotein in the human kallikrein family with a molecular weight of $33 \mathrm{kD}$, containing $7 \%$ carbohydrate. 
The PSA gene (hKLK3) is a member of a gene family that includes hKLK1, hKLK2, hKLK3 and KLK-L1, all located on chromosome 19 [1]. Secreted from both benign and malignant prostate epithelial cells, PSA's function is to cleave and liquefy the ejaculate [2]. PSA is androgen-dependent and prostate organ-specific, but it is not cancer-specific [1].

Under normal circumstances, only small amounts of PSA circulate in the serum in both uncomplexed (free) and complexed (bound) forms [2] [3]. Elevated serum levels of PSA can occur in association with benign prostatic hyperplasia $(\mathrm{BPH})$ or prostatitis but are more closely associated with localized as well as advanced carcinoma [3].

Since PSA's discovery in 1979, its utility as a biomarker for malignant disease in the prostate, through clinical application in the late 1980's and 1990's, has evolved into an invaluable tool for determining the need for prostate biopsy and, after diagnosis, as both a means of risk-stratification and to monitor response to selected treatment modalities [2].

A serum level of $4 \mathrm{ng} / \mathrm{mL}$ PSA is generally used as the cut-point between normal and abnormal [3]. Despite its utility, PSA testing is not perfect. 20\% $40 \%$ of patients with organ-confined Prostatic Adenocarcinoma will have a PSA value less than $4.0 \mathrm{ng} / \mathrm{mL}$, while the positive predictive value (PPV) of a serum PSA between $4-10 \mathrm{ng} / \mathrm{mL}$ is only approximately $20 \%-30 \%$ [2] [3]. For serum levels of PSA in excess of $10 \mathrm{ng} / \mathrm{mL}$, the PPV increases to upwards of 72\% [2]. In light of this, combined with variations due to age and ethnicity; age-specific and race-specific reference ranges for serum PSA levels have been proposed [2].

Since its clinical introduction in 1986, PSA-based screening for the early diagnosis of Adenocarcinoma of the Prostate has led to increased detection and therefore incidence of disease, decreased age at the time of diagnosis, decreased grade and stage at the time of diagnosis, and decreased cause-specific mortality [2] [5] [6] [7] [8] [9]. Since 1997, there has been an overall threefold increase in the incidence of the disease in the United States, with the median age at diagnosis dropping from 72 in 1986 to 66 in 2011 [10]. In the Prostate, Lung, Colorectal and Ovarian Cancer Screening Trial (PLCO), the proportion of screening-detected cancers diagnosed at clinical stage I or II increased from $94.2 \%$ in round 1 to $98.5 \%$ in round 2, while the proportion with Gleason scores $>7$ decreased from $10.0 \%$ to $6.8 \%$ [11]. Over the same interval, mortality from Prostate Cancer decreased, possibly a result of screening coupled with earlier intervention and improved treatment modalities.

Opinions differ regarding the utility of screening for the early diagnosis of Adenocarcinoma of the Prostate due to the fact that in a significant proportion of men the disease has a relatively indolent course. The discrepancies among guidelines reflect the difficulty in differentiating indolent from aggressive disease, at what age is early diagnosis most beneficial and how much of an effect will early intervention have on mortality. Guidelines discouraging routine screening are associated with declining localized/regional incidence rates for the 
disease as incidence rates of more advanced stage disease increased in a younger population [12]. It is predicted that this trend will ultimately adversely affect mortality rates.

According to multiple studies with relatively longer follow-up, there is a preponderance of evidence in favor of screening, especially in men aged $55-69$ years. Additionally, it appears that patients who are asymptomatic but who are at increased risk of developing Prostate Cancer benefit from screening. Men older than 70 years with a life expectancy $>10$ years should be engaged in discussions regarding the risks versus the benefits of PSA screening prior to embarking on a screening protocol.

There is abroad consensus that screening should begin earlier for men with risk factors such as family history or African-American heritage, particularly if associated with early-onset Prostate Cancer. Recognition of unique genetic factors in patients with early-onset disease coupled with aggressive behavior can help target screening and therapy and thus reduce disease-specific death among younger men.

\section{Epidemiology}

Adenocarcinoma of the Prostate is a disease most frequently diagnosed between the ages of $65-74$ with a median age at diagnosis of 66 [2]. It is the second most commonly diagnosed cancer in men and the third leading cause of cancer death in American males [2] [3]. About 1 man in 7 (14.2\%) will be diagnosed with the disease during his lifetime, and the American cancer society estimates that in 2017 , there were approximately 161,000 new cases and approximately 26,000 deaths [2].

$60 \%$ of cases are diagnosed in men aged 65 and older; and about 1 of 39 (2.65\%) will die from the disease [3]. Approximately $10 \%$ of cases represent early-onset Prostate Cancer, defined as diagnosis at the age of 55 or less [5].

The prevalence of Adenocarcinoma of the Prostate increases rapidly with age, and unlike most other cancers which have a peak age of incidence, disease incidence continues to increase with advancing age [2]. Autopsy studies have demonstrated that in Western-European and American men, the prevalence of disease in the 40 to 50 -year age range is about $20 \%-30 \%$ and that approximately one third of men aged 50 to 60 will have histologically evident prostatic cancer at autopsy with the percentage increasing to approximately $75 \%$ in men older than 85 years of age [13] [14]. Although Prostatic Adenocarcinoma has no national or ethnic boundaries, its prevalence is highest among American men [4]. Breslow et al. demonstrated that on average about $15 \%$ of men aged 45 - 54 years have latent carcinoma with a prevalence ranging from $9 \%$ to $22 \%$, depending on the geographical distribution of the study population [10].

Age-adjusted mortality rates decreased in the U.S by $1.2 \%$ between 1991 and 1994, and by 4.5\% between 1994 and 1999 [16]. The average age of death from Adenocarcinoma of the Prostate from 2003-2007 was 80 years and $71 \%$ of those 
deaths were in men older than 75 years [17]. During these years, as mortality declined, average life expectancy for males increased, meaning that mortality from Prostate Cancer would have been expected to rise, which it did not. It has been hypothesized that this might be due to a combination of screening and improved treatment [2] [5] [7] [18]. Conversely, it has been argued that decreased mortality rates have been associated with significant rates of diagnosis of clinically insignificant disease and therefore over-treatment, with its attended morbidity, particularly as the number of deaths annually due to Prostatic Adenocarcinoma appears to be proportionally outweighed by the number of newdiagnoses [2].

\section{Anatomy and Pathophysiology}

The prostate gland is an integral part of the male reproductive system and helps both to elaborate and store seminal fluid. It contains glandular epithelial elements that produce $20 \%$ of the fluid constituting semen in order to provide a suitable environment for survival and function of spermatozoa [1]. Components of prostatic secretions include: citric acid, fructose, polyamines, phosphoryl choline, prostaglandins, zinc and secretory proteins. Several prostatic secretory proteins are found in abundance and have clinical significance [1] [4] [10]. These include: PSA (human kallikrein 3 [hK3, protein; or KLK gene]) which is necessary for the liquefaction of ejaculate, human kallikrein 2 (hK2 or KLK2), a trypsin-like enzyme which carries out cleavage at arginine residues and is actually more potent than PSA, prostase/KLK-L1, another serine protease in the human kallikrein family, prostatic acid phosphatase (PAP), which acts to hydrolyze organic monophosphate esters to yield inorganic phosphate and alcohol, prostate-specific protein (PSP-94)/B-microseminoprotein (B-MSP), which acts to inhibit follicle stimulating hormone (FSH), there by affecting the quality of sperm structure and function, and Zinc-a-glycoprotein, a protein regulated by glucocorticoids which affects fertilization and lipid mobilization [1].

In the average adult male, the prostate is $3 \mathrm{~cm}$ long, $4 \mathrm{~cm}$ wide, $2 \mathrm{~cm}$ in AP depth and weighs about 20 grams [19]. It surrounds the prostatic urethra, and because of this location, prostate disease will often effect urination and/or ejaculation [19]. Its glandular component is approximately two thirds of its volume while the other third is fibromuscular in nature [20]. Three distinct zones are identified in the prostate: the peripheral, central, and transition zones account for $70 \%, 25 \%$ and $5 \%$ of the prostate volume respectively [1] [2] [3] [20]. These areas each have distinct ductal systems and are differentially afflicted by neoplastic processes.

$60 \%-70 \%$ of Prostatic Adenocarcinomas originate in the peripheral zone, $10 \%-20 \%$ in the transitional zone and $5 \%-10 \%$ in the central zone. Of the cases that arise in peripheral zone, most arise in a classically posterior location where they may often be palpated on digital rectal exam (DRE).

$15 \%$ or so of radical prostatectomy specimens reveal predominantly anteriorly based tumors, some in the transition zone, with others arising in the anterior 
horn of the peripheral zone [10].

Peripherally located adenocarcinomas of the prostate tend to extend via peri-neural space invasion, and extra-prostatic extension preferentially occurs posteriorly and posterolaterally, commonly involving the seminal vesicles, and even the base of the urinary bladder, which in more advanced disease may result in ureteral obstruction [1] [3]. The most frequent sites of metastases are lymph nodes, first in the pelvic distribution and then retroperitoneal, and bones, particularly the vertebral bodies and ribs, followed by the long bones and skull. Lung metastases from metastatic Prostatic Adenocarcinoma are not uncommon [1]. Late in its course, the disease will also spread to other organs, commonly the liver and adrenalgland [21].

Prostate gland differentiation and function, as well as Adenocarcinoma of the Prostate growth and progression, are critically dependent upon Androgen Receptor (AR) signaling [1] [19] [22]. Androgens include testosterone, made in the Leydig cells of the testes, Di Hydro Epi Androstenedione (DHEA), an intermediate product of steroidogenesis, and Di Hydro Testosterone (DHT), the active metabolite of testosterone within the prostatic epithelial cell [1], as testosterone itself is transported into the cell cytoplasm where it is converted to DHT by the enzyme 5-alpha-reductase [19] [22].

DHT has 10 times the binding affinity for the AR in the prostate than testosterone [22]. DHT transactivation of AR involves several coregulatory proteins that respond differently to a changing microenvironment in order to target specific genes involved in cell growth, phosphorylation and transportation of ATP [1] [22]. Further, DHT binding promotes recruitment of protein kinases, resulting in phosphorylation of serine residues which serve to protect them from proteolytic degradation, stabilization, and transcriptional activation.

Normal prostatic epithelium maintains a balance between the rate of cell proliferation and the rate of apoptosis. In Prostate Cancer, this balance is lost first through the breakdown of cellular interactions between the extracellular matrix and basement membrane, and subsequently by induction of malignant transformation due to up-regulation of non-histone proteins such as High-Mobility Group (HMG) proteins which promote increased gene transcription, leading to tumor growth [1] [22].

The importance of androgens in the growth and spread of Prostatic Adenocarcinoma is demonstrable in the disease regression, albeit temporarily, seen following castration or the use of anti-androgens [1] [2] [3] [19] [22]. Further, the Prostate Cancer Prevention Trial (PCPT) and the Reduction by Dutasteride of Prostate Cancer Events (REDUCE) trials, demonstrated that inhibition of the conversion of testosterone to the more potent DHT by a 5a-reductase inhibitor reduces the incidence of well-differentiated Prostatic Adenocarcinoma by $25 \%$ $30 \%$ [1] [13] [18]. Finally, androgen receptor biology plays a central role in castrate-resistant disease [1] [13].

More than $95 \%$ of prostate carcinomas are adenocarcinomas. Prostatic Intraepithelial Neoplasia (PIN) and Atypical Small Acinar Proliferation (ASAP) are 
thought to be precursor lesions [2]. PIN consists of architecturally benign prostatic acini or ducts lined by cytologically atypical cells, and is sub classified into Low-Grade PIN (LGPIN) and High-Grade PIN (HGPIN) [1]. Evidence that HGPIN is a precursor to some prostate carcinomas includes an increase in the size and number of HGPIN foci in patients with adenocarcinoma compared to those without carcinoma, and, as HGPIN volumes increase, there occurs a greater number of multifocal carcinomas. Further, biomarkers and molecular changes in HGPIN demonstrate similarities to carcinoma.1However, PIN does not need to be present for carcinoma to arise; low grade carcinomas, especially those present within the prostate transition zone, are not closely related toHGPIN [1].

ASAP represents a higher risk histology than PIN and is suggestive of, but not diagnostic for, carcinoma [2]. Its presence represents an indication for recommendation for repeat biopsy.1The likelihood of cancer after a pathologic recognition of ASAP is $40 \%-50 \%$ [1].

In men with a previously atypical biopsy, the level of serum PSA elevation or findings on DRE do not correlate well with the risk of subsequent positive biopsy [1]. The histology for the remaining $5 \%$ of carcinoma of the prostate is heterogeneous, arising from stromal, epithelial or ectopic cells. Non-adenocarcinoma variants are classified as epithelial ornon-epithelial [1] [2] [10].

\section{Molecular Biomarkers}

PSA has been used as a screening tool, a means of risk-stratifying known Adenocarcinoma of the Prostate and for following the response of the disease to selected treatment modalities since the early 1990s. Use of PSA-based screening became ubiquitous in the US in 1992 after studies demonstrated that screening increased the detection of Prostate Cancer [5]. However, its use is complicated by the fact that while PSA is prostate specific, it is not specific tocancer [3], and therefore only about $25 \%$ of men with PSA in the $4-10 \mathrm{ng} / \mathrm{mL}$ will be found to have the disease [1] [2]. Further, its utility is also compromised by the fact that other conditions such as Benign Prostatic Hyperplasia (BPH), prostatitis, ejaculation, as well as urethral instrumentation may elevate PSA, producing false positive results [2].

A "normal" serum PSA has traditionally been defined as $<4 \mathrm{ng} / \mathrm{mL}$, although lately this is often stratified by age, with an increased upper range seen in older populations and age-specific reference ranges have been proposed [1] [2]. Generally speaking, the Positive Predictive Value (PPV) of a serum PSA between 4 and $10 \mathrm{ng} / \mathrm{mL}$ is approximately $20 \%-30 \%$ [1] [2]. For levels in excess of 10 $\mathrm{ng} / \mathrm{mL}$, the PPV increases to as high as $71.4 \%$ [1] [2]. However, the results of the PCPT, which included biopsy regardless of PSA level-thereby avoiding bias-demonstrated that there is no level of PSA below which Adenocarcinoma of the Prostate risk falls to zero [6]. PSA thus seems to be indicative of a continuum of risk-the higher the level, the higher the risk. 
Screening and detection strategies for Prostate Cancer therefore must take into consideration risk factors other than PSA, such as family history, ethnicity, and race [1] [2]. Risk calculators integrating these variables have been designed to determine an individual's risk of not only having Prostate Cancer but their risk of having a high-grade, more aggressive carcinoma [1] [2]. As 5a-reductase inhibitors lower the PSA by approximately 50\%, thereby establishing a new baseline PSA, their historical use by a patient must be considered as well. Interestingly, serum PSA levels have also been noted to be decreased in men with high Body Mass Indices (BMI) compared with normal weight men, possibly as a result of hemodilution [1] [2].

Numerous strategies to refine PSA testing for cancer detection have been explored. PSA Velocity (PSAV) utilizes the change in PSA value over time, a more rapid rise being considered a more ominous finding. The use of PSA Density (PSAD) attempts to standardize the ratio of PSA to prostate volumes, higher levels being more indicative of a need for biopsy [1].

Generally, only a small proportion of active PSA diffuses into the circulation, where it is rapidly bound or complexed by covalent attachment to protease inhibitors. Inactive PSA can also enter the bloodstream, where it circulates in an unbound state as free-PSA (fPSA) [1]. The use of these PSA isoforms is accomplished by calculating the ratio of percent of inactive or unbound PSA (fPSA) to total-PSA (tPSA). As this drops below 20\% the patient's risk of harboring Prostatic Adenocarcinoma rises, indicating a need for biopsy [1].

Several PSA isoforms have been identified that further increase the diagnostic specificity for Adenocarcinoma of the Prostate [23]. In particular, the [-2] form of pro-PSA has shown improved performance over either total or free PSA for detection of cancer on biopsy [23]. Prostate Health Index (PHI), a PSA based test, has recently been approved in the US. PHI is a mathematical formula that utilizes tPSA, fPSA and [-2] pro-PSA [23] by combining all three forms into a single score calculated by using the following formula: ([-2] pro-PSA/free PSA) $x$ $\checkmark$ PSA. Men with a higher tPSA and pro-PSA with a lower fPSA, and therefore a higher PHI, are more likely to have clinically significant disease [23].

Among 892 men with tPSA serum levels from $2-10 \mathrm{ng} / \mathrm{mL}$ and normal DRE who underwent prostate biopsy; the mean PHI scores were 34 for men with negative biopsies and 49 for men with positive biopsies. Sensitivity for this test was therefore found to be in the $80 \%-95 \%$ range.

The $4 \mathrm{~K}$ Score $(4 \mathrm{~K})$ is a novel blood test that incorporates a panel of four kallikrein related markers: the tPSA, fPSA, intact-PSA (iPSA), and human kallikrein-related peptidase 2 (hK2 Protein), which is a serine protease closely related to PSA [24]. Physiologically, hK2 cleaves pro-PSA to generate the enzymatically active form of PSA in the prostate. Immunohistochemical studies have demonstrated hK2 to have an increased expression as the promoted tissue evolves from normal to poorly differentiated prostatic epithelium [25].

$4 \mathrm{~K}$ was studied in an independent cohort of 2914 previously unscreened men in the first round of the Rotterdam arm of the ERSPC [24]. The study design 
provided 728 participants in the training set and 2186 in the validation set [24]. Application of $4 \mathrm{~K}$ to 1000 men with elevated PSA resulted in 513 men avoiding biopsy while missing only 12 of 100 Gleason score $\geq 7$ adenocarcinomas. Results from these studies consistently show that $4 \mathrm{~K}$ can effectively identify high-grade diseases and, when used in concert with other clinical information analytics, can provide a predictive risk stratification for particular individuals in terms of who, if biopsied, will be found to harbor a high-grade Gleason score 7 or greater Adenocarcinoma of the Prostate, thereby reducing the number of unnecessary biopsies by as much as $49 \%-57 \%$ among men undergoing screening [24].

Prostate Cancer Gene 3 (PCA3) elaborates a non-coding prostate-specific messenger RNA (mRNA) which is over expressed in cancerous tissue compared to benign tissue activity and may be measured on a urine assay following prostate exam [26] [27]. There is a median 66-fold up regulation of PCA3 in Prostatic Adenocarcinoma when compared with adjacent non-cancerous tissue [2]. The presence of elevated levels of PCA3 in urine has a positive predictive value for the presence of cancer on prostate biopsy with an accuracy of $74.6 \%$ and is particularly useful in the evaluation of men with a negative prior biopsy and a rising PSA [2].

In a comparison study of PHI, PCA3 and the urinary TMPRSS2: ERG test, PHI and PCA3 possessed statistically similar areas under the curve on ROC statistical analysis as predictive of the presence of Prostate Cancer [23] [28]. In 658 men with PSA levels of $4-10 \mathrm{ng} / \mathrm{mL}$ from the study population, there was significant correlation between PHI and Gleason score on positive biopsies, whereas PCA3 and TMPRSS2: ERG were found to be independent of Gleason Score [23] [28].

The common goal of these tests is to decrease the number of false-positive test results, thus increasing the specificity and positive predictive value of testing and thereby lead to fewer unnecessary biopsies, lower costs and reduce associated morbidity [1] [2] [4] [6].

\section{Risk Factors}

Several risk factors for Adenocarcinoma of the Prostate have been identified, and while age, elevated BMI, race, family history, hormone levels, environmental and genetic influences are all suspected to play a role in the development of disease, the only well-established risk factors are age, race/ethnicity, and family history of the disease.14While each individual risk factor is linked to specific associated increases in risk, it is likely that development of disease is due to the concurrent influence of multiple factors.

\subsection{Age Associated Risk}

Autopsy studies have demonstrated that the prevalence of Adenocarcinoma of the Prostate increases with age, with a disease prevalence of approximately $15 \%$ in men 45 - 54 years, $33 \%$ in men 40 - 60 years, and greater than $75 \%$ in men 
aged 85 years or older [6] [10]. Since the introduction of PSA testing, there has been a trend toward a greater number of younger men being diagnosed with Prostate Cancer, the median age at diagnosis being shifted from age 70 to age 66 [14].

In men age 80 and older the incidence rate in 2005 was 0.56 relative to 1985 while in men between the ages of 50 - 59 the incidence rate was 3.64 [14]. The incidence of Adenocarcinoma of the Prostate in men under the age of 55 years increased from 5.6 in 1986 to 32 cases per 100,000 person years in 2008, a greater increase in incidence than any other age group [5]. This is an especially important concern given that existing data suggests that survival with this carcinoma varies by age at diagnosis, being more aggressive in younger men among whom those with a higher Gleason Grade or locally advanced disease at the time of diagnosis will have a particularly poor prognosis [5]. Specifically, men diagnosed with stage IV cancer or Gleason score of $8-10$ and aged $<55$ years had a 1.4 to 1.5 -fold greater risk of dying of their cancer compared to men aged 65 - 74 years [5] [29].

\subsection{Ethnicity}

Race appears to be both a risk factor and a prognostic factor for Adenocarcinoma of the Prostate, and African-American men carry an occurrence risk that is 1.7 times above that of Caucasians while their risk of dying of the disease is 2.3 times greater [14]. The highest reported incidence rate for prostate carcinoma occurs among African-American and Jamaican men of African origin [10]. African-American men are also at a higher risk of presenting with more advanced stage at an age 2 - 3 years earlier than Caucasian men [10]. Analysis of 2014 SEER data demonstrated that African-American men at the age of $40-49$ years had higher Gleason score and 2.8 - 3.2 times higher mortality and metastatic disease compared with American men of European descent of the same age group [10].

\subsection{Familial Clustering}

Familial clustering of Adenocarcinoma of the Prostate appears to be the strongest determinant of risk for development of disease [5] [10] [17] [19]. Genetic studies suggest that strong familial predisposition may be responsible for between $5 \%$ and $10 \%$ of prostatic carcinomas [5]. Men whose first-degree family members have Prostate Cancer appear to possess double the risk of developing the disease compared to individuals with a negative family history, and this risk appears to be greater for men with an affected brother than for men with an affected father [19].

Age of onset in the family member with Adenocarcinoma of the Prostate also affects relative risk (RR) [2]. If the age of onset in the affected family member is 70 years of age or greater, the patient's RR is increased fourfold. If the age of onset is between 60 and 70 years, the RR is increased fivefold; and if the age of on- 
set is below 60 years, the RR is increased sevenfold [2]. For example, if the patient's father had been diagnosed prior to 60 years of age, the RR for that patient would increase from 1.5 to 2.5. The RR further increases to 4 times if the patient has two or more affected first-degree relatives, diagnosed at any age $(R R=4.39)$ [10].

Familial Adenocarcinoma of the Prostate tends to manifest 6 - 7 years earlier than sporadic disease [10]. In the Health Professionals Follow-up Study, having a positive family history was associated with a greater increase in risk among men under 65 years $(R R=2.3)$ than among their counterparts aged 65 years and over $(\mathrm{RR}=1.7)$ [5]. Additionally, twin studies have demonstrated that the heritable component of risk is over $40 \%$ [1].

\subsection{Susceptibility Genes}

Men diagnosed with Adenocarcinoma of the Prostate prior to age 55 are also more likely to carry a greater number of genetic variants with small-to-moderate penetrance that have been found to have increased their risk in Genome Wide Association Studies (GWAS) [5] [10].

Hereditary Prostate Cancer Gene 1 (HPCG1) mutations and inherited mutations in other genes such as Breast Cancer Susceptibility Genes (BRCA1, BRCA2, and HOXB13) account for some cases of hereditary disease [5]. Men with BRCA2 or HOXB13 gene mutations may have a high risk of developing more aggressive, life-threatening Prostate Cancer [5].

Approximately $15 \%$ of Adenocarcinoma of the Prostate is likely to be due to germline factors [7]. GWAS focusing on men under the age of 55 with early-onset disease have revealed several Single Nucleotide Polymorphisms (SNPs) in the germline DNA associated with an increased risk in these populations [5] [10] [30] [31].

\section{1) BRCA Mutations}

Female carriers of the BRCA1 and 2 mutations are at risk for developing breast and ovarian carcinomas and these mutations predispose male carriers to the development of Adenocarcinoma of the Prostate [10] [32]. While there is some controversy regarding the level of increased risk to male BRCA1 carriers, they appear to be at $1.8-4.5 \mathrm{RR}$ of developing Prostate Cancer at $<65$ years of age [10]. BRCA2 is associated with RR of 2.3 in men $<65$ years of age [7]. In a study of 263 men with known BRCA mutation, BRCA2 carriers had an estimated 23-fold increased risk of developing Prostatic Adenocarcinoma prior to age 55 years as compared to age-matched controls [10]. Further, all studies have consistently reported that BRCA2 carriers who develop Prostate Cancer, present with aggressive disease, characterized by high rates of lymph node involvement, distant metastasis at diagnosis and a higher mortality rate as compared with non-carriers [10].

These mutations may be tested for, but that testing should be indicated. The strongest indication for a need for testing for germline mutation of BRCA genes 
in patients with Adenocarcinoma of the Prostate is early-onset in the face of a family history of prostate, breast, and/or ovarian cancers. Nevertheless, this mutation's overall contribution to early-onset disease appears to be limited as BRCA2 mutation is only found in $2 \%$ of cases diagnosed under the age of 55 years [1] [5] [10] [30].

Hypermethylation of glutathione S-transferase (GSTP1) gene, which downregulates GSTP1 expression, is the most common somatic mutation found in Adenocarcinoma of the Prostate [13]. These mutations give rise to chromosomal rearrangements which juxtapose the coding sequence of an ETS family transcription factor gene next to the androgen-regulated TMPRSS2 promotor resulting in epigenetic alterations [13]. Epigenetic modifications in a subset of prostatic carcinomas also appear to render ineffective a number of other tumor suppressor genes including: PTEN, RB, p16/ink4a, MLH1, MSH2, and APC [13]. Genes linked to this activity include the hereditary Prostate Cancer Gene 1 (HPCG1), the androgen receptor and the vitamin D receptor [19].

\section{2) HOXB13 Gene Mutations}

A positive family history of Adenocarcinoma of the Prostate associated with increased risk for early-onset, relatively indolent disease, may be due to a rare non-synonymous Single Nucleotide Polymorphism (SNP) mutation in the first exon coding region of the Homeobox B13 Gene [HOXB13 (G843 or rs138213197)] mapping to an area of interest at 17q21-q22, having been identified by GWAS [10]. This mutation was found to be present in $1.4 \%$ of cases of Prostate Cancer compared to only $0.1 \%$ of controls and was significantly more common in men with early-onset, familial Prostatic Adenocarcinoma (3.1\%) than in those with late-onset, sporadic disease (0.6\%) [1] [5] [10] [31]. HOXB13 is a transcription factor important in early prostatic development and differentiation by its interaction with androgen receptors to promote development and differentiation of prostate cells [10]. The mutated form is postulated to act as an oncogene in androgen-independent cancerous cell lines through its activity in disrupting the normal activities of the AR in both hereditary and sporadic cases, but the exact mechanism of HOXB13's role in carcinogenesis remains unknown for the time being [5] [10] [31].

\section{3) Other}

Chromosomal rearrangements, or copy number abnormalities, at $8 \mathrm{p}, 10 \mathrm{p}$, 11q, 13q, 16q,17p, and 18q have been described in Prostate Cancer [1] [2] [19] [22]. In 2005, Tomlins, et al. discovered the presence of gene fusions in Adenocarcinoma of the Prostate, stimulating work to determine their potential utility as biomarkers.33Subsequently, fusions of the 5' untranslated region of the androgen-regulated Transmembrane Protease, Serine 2 (TMPRSS2) with Virus-Erythroblastosis Transformation Specific (V-ETS) E6 oncogene homolog (ERG) or V-ETS variant 1 (ETV1) have been found to be nearly $100 \%$ specific for Prostatic Adenocarcinoma and present in at least 50\% of PSA-screened adenocarcinomas of the prostate. Both ERG and ETV1 are members of the E26 
V-ETS transcription factor family, and TMPRSS2: ERG fusions represent about 90\% of all ETS gene fusions [33] [34].

Two transcription factors, ERG and ETV1, have also been found to be overexpressed in prostatic carcinoma tissue.33Additionally, TMPRSS2 was found to be fused to these genes suggesting that fusion may have accounted for their overexpression [33]. This genetic rearrangement occurs in approximately $50 \%$ of prostatic tumors and is postulated to represent an early molecular event in carcinogenesis, and furthermore, may yield a distinct phenotype with a more aggressive natural history independent of Gleasongrade [33] [34] [35].

\section{Risk Groups}

Contemporary treatment patterns for Adenocarcinoma of the Prostate are marked by both over-treatment of low-risk disease and under-treatment of high-risk disease. A key approach to mitigating this problem is an attempt to develop better and more consistent risk stratification intended to help identify the best timing and intensity of treatment for a given patient.

\subsection{American Urologic Association}

A three-level group classification has been adopted by the American Urological Association (AUA) for stratifying the risk localized Adenocarcinoma of the Prostate: [2]

1) Low risk $=$ PSA $<10 \mathrm{ng} / \mathrm{mL}$, Gleason $<6$, and clinical stage $\mathrm{T} 1$ or $\mathrm{T} 2 \mathrm{a}$

2) Intermediate risk $=$ PSA $10-20 \mathrm{ng} / \mathrm{mL}$, Gleason 7 , or clinical stage $\mathrm{T} 2 \mathrm{~b}$

3) High risk $=$ PSA $>20 \mathrm{ng} / \mathrm{mL}$, Gleason $8-10$, or clinical stage T2c, or T3a

Despite its simplicity, overall utility and wide usage, this system has some drawbacks in that it tends to overweigh the prognostic value of $\mathrm{T}$ stage which is not always an accurate measurement of tumor extent within the T2 category, and it does a poor job of distinguishing between Gleason $3+4$ and Gleason $4+3$ which behave very differently within the Gleason 7 category [1] [2]. Most importantly, it is not a true multivariable instrument [2]. For example, both a PSA 19.8, Gleason $4+3$, stage T2b tumor and a PSA 4.2, Gleason $3+4$, stage T1c tumor are 'intermediate risk' in this classification but would, in fact, be expected to demonstrate quite different clinicalbehavior [2].

\subsection{CAPRA Score}

The UCSF Cancer of the Prostate Risk Assessment (CAPRA) Score is intended to combine the accuracy of nomograms with the ease of calculation of a risk grouping system [2]. To calculate the CAPRA score, points are assigned based primarily on the PSA and Gleason score, with lesser weights given to T stage, percent of biopsy cores positive, and patient age. Points are added to yield a score. Overall, every 2-point indicates roughly a doubling of long-term risk [2].

1) CAPRA scores of 0 - 2 range indicate relatively low-risk disease

2) CAPRA scores of 3 - 5 range indicate tumors that are intermediate risk 
3) CAPRA scores 6 - 10 range indicate high risk tumors

The CAPRA score has been found to offer better accuracy than competing instruments in an independent head-to-head comparison study [2]. Moreover, it has been shown to predict metastasis and mortality as well as biochemical outcomes [2]. CAPRA score is primarily meant to indicate relative rather than absolute risk [2]. Thus, a tumor with a CAPRA score of 4 has an intermediate risk of recurrence or progression after surgery or radiation. This tumor will be more likely to progress than one with a score of 2 and less likely to progress than one with a score of 6 regardless of treatment approach or setting [2].

\section{Adenocarcinoma of the Prostate}

\subsection{Symptoms}

Early in its course, Adenocarcinoma of the Prostate generally causes few, if any, specific symptoms, and a significant percentage of patients with low stage disease are asymptomatic [1] [2] [19]. Stranguria (slow stream), nocturia (nighttime voiding), and increased urinary frequency are common symptoms associated with an enlarging prostate gland but often are unrelated to the presence of Adenocarcinoma [1] [2].

The presence of symptomatic may suggest locally advanced and/or metastatic disease [1] [2] [19]. Manifestations of locally advanced Adenocarcinoma of the Prostate include obstructive urinary symptoms from growth of the tumor into the urethra, bladder neck, ureteral obstruction secondary to invasion of the bladder trigone, causing hydronephrosis, renal insufficiency or renal failure, hematospermia or decreased ejaculate volume, and rarely, impotence [1] [2]. Commonly, such symptoms are attributable to coexisting BPH.

Manifestations of metastatic disease may include bone pain and pathologic fractures as a result of expansile osteoblastic lesions, anemia secondary to bone marrow replacement, lower extremity edema due to lymphatic obstruction, retroperitoneal fibrosis from reaction to lymph node metastases, paraneoplastic syndromes associated with brain, liver or adrenal metastases, disseminated intravascular coagulation as a result of sepsis secondary to urinary obstruction, and paresthesias, weakness of the lower extremities, urinary or fecal incontinence and even paralysis due to spinal metastases and cord compression [1] [2] [19]. It is for these reasons that early detection has been deemed imperative in order to identify Adenocarcinoma of the Prostate while it remains confined to the prostate.

\subsection{Diagnostic Evaluation}

A physical exam, including an assessment for lower extremity lymphedema and a Digital Rectal Exam (DRE) with special attention to the prostate, as well as a focused neurologic exam, specifically to assess the bulbocavernosus reflex and function of the lower extremities should be performed on all patients at risk for Adenocarcinoma of the Prostate [1] [2] [19]. The size and consistency of the 
prostate should be noted, even though prostate size as determined by DRE, does not correlate well with severity of symptoms or degree of obstruction [1] [2].

BPH usually results in a smooth, firm, elastic enlargement of the prostate. Induration, nodules, grittiness or even hard areas of the prostate, if detected must alert the physician to the possibility of carcinoma and the need for further evaluation, i.e. PSA testing, Transrectal Ultrasound (TRUS), and biopsy [1] [2] [19]. A urinalysis to exclude infection, hematuria or significant proteinuria is required. Elevations in serum creatinine levels as an indication of renal insufficiency may be observed in $10 \%$ of patients with prostatism and warrants measurement of the patient's post void residual urine volume, as well as upper-tract imaging to rule out obstruction at the level of the bladder outlet or above [1] [2] [19].

Most physicians will include PSA testing in the patient's initial evaluation, particularly if their presentation includes irritative or obstructive symptomatology. PSA testing, compared with DRE alone increases the ability to detect Prostate Cancer, but because there is much overlap between levels seen in BPH and Adenocarcinoma of the Prostate, particularly in the $4 \mathrm{ng} / \mathrm{dL}$ to $10 \mathrm{ng} / \mathrm{dL}$ range, its use as a screening tool remains controversial [1] [2] [19]. A multi-center screening study of 6630 men, reported a detection rate of $3.2 \%$ for DRE, $4.6 \%$ for PSA and 5.8\% for the two methods combined [36]. TRUS and/or cystoscopy are useful in determining prostate size for men planning to undergo intervention in order to best target the appropriate procedure. If the patient has significant microscopic hematuria (>3 RBC per high powered field) or gross hematuria, then cystoscopy and upper tract imaging are mandatory to rule out other bladder or renal pathology [1] [2] [19].

Measurement of flow rate and determination of post-void residual urine are helpful in determining the degree of bladder outlet obstruction. Pressure-flow studies and urodynamic profiles are useful in patients with suspected neurologic disease or those who have failed intervention for bladder outlet obstruction and may be predictive in determining the likelihood for resolution versus persistence of irritative voiding patterns [1] [2].

\subsection{Prostate Biopsy}

Prostate biopsy should be considered in men with a persistently elevated serum PSA, abnormal DRE, or a combination of the two taking into account comorbidities, life expectancy and, levels of anxiety or risk aversion; and is generally performed under TRUS guidance using a spring-loaded biopsy device coupled to the imaging probe [1] [2].

Biopsies are taken throughout the peripheral zone of the prostate with optional additional sampling of any abnormal areas on DRE and/or TRUS [1] [2]. Traditionally, six biopsies were taken along a parasagittal line between the lateral edge and the midline of the prostate at the apex, mid-glad and base bilaterally [1] [2]. However, several investigations have demonstrated that increasing the 
number to $>10$ biopsy cores and performing more laterally directed biopsies of the peripheral zone increased detection rates $14 \%-20 \%$ over the more traditional technique and most Urologic Specialists now take sextant biopsies of each lobe of the prostate, for a total of 12 cores [1] [2].

Although a small number of adenocarcinomas of the prostate will originate in the transitional zone, specific transitional zone biopsies add little to overall cancer detection rates when an extended pattern biopsy is performed [1] [2]. There is ongoing interest in the use of even more extended biopsy schemes, referred to as saturation biopsy techniques, using either the traditional trans-rectal approach or, alternatively, trans-perineal, to improve cancer detection, usually in those who have had a negative biopsy but are thought to be at an increased risk of Adenocarcinoma of the Prostate based on a persistently abnormal serum PSA [1] [2].

MRI of the prostate has demonstrated increased detection rates during directed biopsies accomplished when overlaying the MRI images on real-time TRUS images and has been found to accurately detect clinically significant Prostatic Adenocarcinoma upon comparison to radical prostatectomy histology [37] [38]. Moore, et al. reported that in 599 biopsy-naïve men 374 (63\%) had suspicious findings on MRI and 248 (66\%) of these were found to have Adenocarcinoma of the Prostate on directed biopsy.37In this group of men, standard biopsy approach reported a detection rate of only $50 \%$ [37].

MRI/US fusion biopsy has been reported to detect Prostate Cancer in 34\% $37 \%$ of patients with prior negative TRUS biopsies, with one third of these patients harboring high-grade cancer, defined as Gleason score of $\geq 8.38$

It is hoped that MRI/US-fusion guided, targeted biopsies will provide widespread improvement in the detection of clinically significant lesions and lead to a decrease in the number of biopsies in men with indolent, clinically insignificant disease [38].

\subsection{Grading and Staging}

The Gleason system is the most commonly employed grading system for Prostate Cancer [2]. Using the lower-power microscopic appearance, a primary grade based on the degree of anaplasia and disruption of normal glandular architecture is assigned to the pattern of cancer that is most commonly observed and a secondary grade to the second most commonly observed pattern in the specimen [1] [2].

Gleason Grades range from 1, for the most well-differentiated tumors to 5 for the most anaplastic tumors. Scores are obtained by adding the two grades.

Gleason patterns 1 and 2-rarely assigned, and have come to be viewed by some as variations of benign disease [1] [2] [3].

Gleason pattern 3-lower-grade disease of variable sized glands that percolate through normal stroma and between normal glands.

Gleason pattern 4-intermediate to high-grade disease made of incompletely 
formed glands with variable amounts of fusion and more infiltrative growth pattern.

Gleason pattern 5-high-grade disease with predominate findings of single infiltrating cells with no gland formation [1] [2].

A total Gleason score of $6(3+3)$ tumor is regarded as relatively more indolent disease. In differentiating intermediate from high-grade tumors, the primary Gleason pattern is the most important determinant of biologic risk. Thus, among Gleason score 7 tumors, those assigned $4+3$ are regarded as being more aggressive than $3+4$ [1] [2].

Age at diagnosis is also a well-recognized prognostic indicator. High grade and/or advanced stage disease in young men is often more aggressive, than in older men.10The clinician's task is thus not only to establish a diagnosis of Adenocarcinoma of the Prostate but to differentiate aggressive from indolent disease.17Therefore, screening programs have come to utilize the most prominent risk factors to more accurately identify men more likely to harbor occult disease and thereby enable physicians to judiciously test those patients who would most benefit from early detection [39].

\subsection{Studies}

There are a number of factors which have been identified that increase the risk of developing Adenocarcinoma of the Prostate at an earlier age, each with its own unique contribution to the individual's relative risk, and although it has most often been considered a disease of the elderly (>age 65), its occurrence is not uncommonin men younger than age 55 [3]. Men diagnosed at an early age often have associated risk factors and present with aggressive disease [10].

Early detection is therefore critical in younger patients and although there is some disagreement among the recommended guidelines regarding PSA-based testing, the fact is that in the decades since the implementation of PSA-based screening, the age-adjusted incidence rate of detection of Adenocarcinoma of the Prostate increased to 237.4 per 100,000, nearly doubling the rate of 1986 [10] [12] [13]. Additionally, the average age of diagnosis decreased from 72 in 1986 to 66 in 2011,accompanied by a 40\% decrease in mortality [1] [10].

The PLCO Cancer Screening Trial randomized 76,693 men in the United States for annual PSA screening or usual care, which, often included PSA and DRE [40]. Men ages 55 - 74 who were randomly assigned to the intervention arm were screened with annual PSA tests for 6 years and DRE for 4 years. A PSA value above $4.0 \mathrm{ng} / \mathrm{mL}$ or an abnormal DRE were indications for biopsy. Approximately $92 \%$ of study participants were followed out to 10 years and $57 \%$ out to 13 years [40].

Over the course of the study, the proportion of cancers diagnosed at clinical stage I or II increased from $94.2 \%$ of tumors to $98.5 \%$, while the proportion with Gleason scores $\geq 7$ decreased from $10 \%$ to $6.8 \%$ [41]. The risk of Adenocarcinoma of the Prostate death after 7 years of follow up demonstrated no statisti- 
cally significant reduction in screening vs control groups [42]. After 13 years of follow-up, the cumulative mortality rates from prostatic cancer in the intervention and control arms were 3.7 deaths and 3.4 deaths per 10,000 person-years, respectively and did not obtain statistical significance [40] [42]. These results are felt to be due not only to the short duration of the study given the natural history of Prostate Cancer, but that almost $40 \%$ of men in the control arm had been screened with PSA outside the parameters of the study, and, finally, that the rate of prostate biopsy among men who crossed the predefined PSA threshold of 4.0 was low [42].

The European Randomized Study of Screening for Adenocarcinoma of the Prostate (ERSPC) randomized 162,387 men in seven countries to PSA screening at 2 or 4-year intervals, using age-adjusted biopsy thresholds ranging from 2.5 to $4.0 \mathrm{ng} / \mathrm{mL}$. With longer follow-up than PLCO and much less contamination among control patients, the study demonstrated screening to be associated with a 20\% relative reduction in Prostate Cancer-specific mortality at 9 years' median follow up [43]. With adjustment for screening compliance, the reduction in mortality rose to $31 \%$ while the hazard curves began to diverge approximately 7 years into the trial [41] [43].

After 11 years of follow-up the ERSPC found that the Prostate Cancermortality ratio was 0.79 in favor of screening [6]. A sub-analysis of patients from one of the ERSPC study centers with particularly high compliance and longer (14 year) follow-up confirmed a reduction in mortality of nearly $50 \%$, which would seem to strongly favor screening for Adenocarcinoma of the Prostate [4] [12] [43]. It is felt that long-term follow-up in both the PLCO and ERSPC trials will prove invaluable in the continued assessment of the effects of PSA-based screening onmortality beyond 10 years and the effects of modified screening methodologies such as the use of percent-free PSA, PHI and $4 \mathrm{~K}$ in their abilities to distinguish between indolent disease and disease that is likely to progress. Studies into the effects of watchful waiting and active surveillance protocols on long term mortality and morbidity compared to the various treatment options would similarly be of great importance.

\subsection{Simulation Models}

Mathematical simulation models have been developed to estimate the number of future Prostate Cancer-specific deaths that might be avoided using programs of early detection and treatment with curative intent [6]. At current screening rates, it is estimated that upwards of 700,000 men would be diagnosed with Prostate Cancer between 2013 and 2025, and that 36,000 - 57,000 disease specific deaths would be prevented.41 Certainly, abandonment of PSA screening would prevent all cases of over-diagnosis but it would also fail to prevent $100 \%$ of avoidable deaths and potentially lead to as much as a $20 \%$ increase in disease-specific deaths [6]. It would likely also lead to an increased incidence of metastatic disease at the time of diagnosis, perhaps to pre-screening levels. Even screening restricted to men under the age of 70 , predicts an additional 13,000 - 
22,000 deaths and an approximate 50\%increase in the incidence of metastatic disease at diagnosis [6].

\subsection{Screening and Early Detection}

Detection of disease at a pre-symptomatic stage is referred to as secondary prevention, and despite the seeming benefits to screening for Adenocarcinoma of the Prostate, its practice has been the subject of substantial ongoing controversy in the United States.

After Prostate Cancer screening with PSA testing became widespread in the early 1990's, the US experienced a $2 \%$ per year increase in the incidence of prostatic adeno carcinoma through 1995, and since then has risen steadily at approximately $1 \%$ annually, even as the median age at diagnosis dropped to 66 in 2011 [4] [5] [12] [16] [39]. Epidemiologic data further demonstrates a $40 \%$ reduction in mortality and a $75 \%$ decline in patients presenting with advanced-stage disease at the time of diagnosis [6].

Currently, out of 1000 age-appropriate men who undergo PSA based screening, 240 of them will have a positive screen, and 100 will go on to have a positive biopsy [44]. Yet $20 \%$ - 59\% of these tumors carry a very low risk of developing metastases or shortening the individual's life span [44]. Nevertheless, 80 of 100 will choose treatment with surgery or radiotherapy, with the net result being that only 3 of the original 100 will avoid metastatic disease and only $1-2$ will avoid a Prostate Cancer-specific death [44]. This feeds the primary argument against screening in that many cancers identified through screening efforts never appear to result in clinically significant disease, a phenomenon referred to as over detection [2].

As a result, in 2012, in order to limit over detection and thus over treatment, the United States Preventive Services Task Force (USPSTF) recommended against PSA-based screening, awarding the test a grade of D based on "convincing evidence that a substantial percentage of men who have asymptomatic cancer detected by PSA screening have a tumor that either will not progress or will progress so slowly that it would have remained asymptomatic for the man's lifetime." [45] [46].

However, practitioners in the field feel that this serves to merely underscore the need for informed consent prior to screening. Such consent must include discussion of all diagnostic and treatment options, as well as potential complications. The case for judicious PSA based screening is supported by the facts that the disease has proven itself burdensome, PSA screening appears to improve detection of clinically important tumors without significant increase in the detection of unimportant tumors, earlier detection through screening has decreased the risk of metastatic disease by approximately three fourths, most PSA-detected tumors are curable, and mortality has declined in regions where screening occurs [1] [4] [11] [13]-[18] [47] [48] [49].

When undertaking screening, the use of both DRE and serum PSA in concert 
is preferable to either one used alone. A study of 6630 men reported a detection rate of $3.2 \%$ for DRE, $4.6 \%$ for PSA, and $5.8 \%$ for the two methods combined [29]. There is also broad consensus that screening should begin earlier for men with risk factors such as family history [2] [10] [17]. Recognition of unique genetic factors in men with early-onset carcinoma and aggressive behavior can help target screening and therapy and thus reduce Prostate Cancer-specific deaths among younger men [7]. Men of African-American ethnicity should also begin screening at an earlier age as aggressive, early-onset Adenocarcinoma of the Prostate is seen more frequently in African-American men than in Caucasian men [1] [2] [10] [47] [48].

Some feel that men with very low serum PSA level $<1 \mathrm{ng} / \mathrm{mL}$ may be able to be screened at less frequent intervals (every $2-3$ years) [6] [48]. Similarly, if the PSA level remains $<1 \mathrm{ng} / \mathrm{mL}$ by the age of 60 , the likelihood of death from Prostate Cancer, by the age of 85 falls to $<1 \%$ [25]. Properly risk-adapted management strategies, including greater use of active surveillance for men with lower risk disease should serve to ameliorate the controversy surrounding screening, and the current American Urological Association (AUA) Guidelines for Early Detection of Adenocarcinoma of the Prostate appear to reflect this: [2].

AUA Guideline 1-Recommends against PSA screening in men under age 40 years (Grade C) [48]. In this age group there is a low prevalence of clinically detectable Prostatic Adenocarcinoma, and there is no evidence to demonstrate any benefit to screening [48].

AUA Guideline 2-Does not recommend routine screening in men between ages 40 - 54 years at average risk (Grade C) [48]. For men, younger than 55 years at higher risk, (positive family history or African-American descent), decisions regarding Adenocarcinoma of the Prostate screening should be individualized and undertaken only after informed consent [48].

AUA Guideline 3-Recommends shared decision-making for men age 55 - 59 years that are considering PSA screening and proceeding based on patient preferences, as the greatest benefit to screening is in this age group [48].

AUA Guideline 4-States that to reduce the harms of screening, a routine interval of two years or more may be preferred over annual screening in those men who have participated in shared decision-making and decided on screening [48]. As compared to annual screening, it is expected that screening intervals of two years preserve the majority of the benefits and reduce over diagnosis and falsepositives [48].

AUA Guideline 5-Does not recommend routine PSA screening in men age $70+$ years, or any individual with less than a 10 - 15-year life expectancy [48].

The National Comprehensive Cancer Network (NCCN) Category A recommendations specify that for patients with a life expectancy $>5$ years or who have symptoms, diagnostic screening should begin by incorporating, a history and physical with attention to risk factors, and include family history, medications, history of prostate disease and any prior screening, including prior PSA and/or 
isoforms, exams and/or biopsies [47]. From that point an assessment of risk vs benefit to any further testing should be discussed with the patient, including, but not limited to their own personal risk factors, if any [47].

Screening should not stop at a baseline PSA, but must include a DRE as well [47]. The NCCN separates patients into two age groups. In men ages $45-75$ years, a PSA $>3 \mathrm{ng} / \mathrm{mL}$ or a very suspicious DRE are indications for biopsy [47]. In men ages $>75$ years, screening should be reserved for selected patients with few if any comorbidities to ensure that intervention would prove to be of benefit [47]. In this setting, a PSA $\geq 4 \mathrm{ng} / \mathrm{mL}$ or a suspicious DRE are indications for biopsy [47].

The NCCN recommendations acknowledges that although screening carries a risk of "over-diagnosis" and that not all men diagnosed with Adenocarcinoma of the Prostate will require treatment, the need for more intense screening practices in at risk populations, such as in African-American men, those with a positive family history of disease and those who live with proven genetic susceptibility [47].

Following the 2012 USPSTF Recommendations against screening for early detection of Adenocarcinoma of the Prostate, rates of PSA screening decreased by up to $10 \%$ among all age groups within most U.S. geographic regions [6]. Rates of prostate biopsy and the incidence of Prostatic Adenocarcinoma declined in unison, with a notable shift towards higher grade, stage and risk upon detection [6].

When PSA testing rates following the 2012 USPSTF recommendation were stratified by age, decreases were noted among all age groups. Reported screening rates declined from $33 \%$ to $25 \%$ for men aged 50 - 59, from $51 \%$ to $43.6 \%$ for men aged 60 - 74 and from $44 \%$ to 37\% for men older than 75 [6]. Frendl et al. analyzed screening data from higher risk patients and found a $25 \%$ decrease in PSA screening in at risk populations following the USPSTF 2012 recommendation [6].

Since the USPSTF 2008 recommendation against PSA screening in men over the age of 75 years and its 2012 overall recommendation against PSA screening at all, SEER data indicate that between 2011 and 2013, the overall incidence of Prostate Cancer declined each year over year in all ages and races while the age-adjusted rate declined from 147.9 per 100,000 men in 2010 to 108 [6]. in2013 [2] [6].

Analysis of stage-specific rates of Adenocarcinoma of the Prostate in men 50 years and older from 2008 to 2012 found that declines occurred in local/regional (early stage) carcinomas which persisted through 2013, but at a slower pace [6] [50]. The National Cancer Database revealed a small increase in clinical stage T3a tumors or higher, from 2011 to 2013as well as a rise in the incidence of metastases at presentation during the same period [6] [50] [51].

Gaylis et al. reported an increase in the rate of positive biopsies from $46 \%$ to $50 \% .51$ Rosenberg and colleagues reported a $29 \%$ increase [8]. Olsson et al. noted 
annual increases, from $39 \%$ in 2010 to $41 \%$ in 2013 to $43 \%$ in 2014 and $46 \%$ in $2015(p<0.001)$ [9]. Gaylis et al. also reported an increase in the proportion of Gleason 8 carcinomas, from $21 \%$ in 2011 to $30 \%$ in 2014 as well as an increase in Gleason 8 - 10 carcinomas [51]. An analysis of all biopsy cores containing Gleason 8 - 10 patterns found an increase from $15 \%$ in 2010 to $25 \%$ in 2015 [9] [51].

\section{Conclusions}

Since 1977, there has been an overall threefold increase in the incidence of Adenocarcinoma of the Prostate [5] [6] [10]. In the US, the median age at diagnosis dropped from 72 in 1986 to 66 in 2011 [5] [6] [10] [18] [47]. Yet, in the face of an increasing life expectancy, mortality from Prostate Cancer declined by some $40 \%$. PSA's introduction and subsequent adoption as a tool in screening for the early detection of Prostate Cancer has been coincident with, and arguably, in large part responsible for, these trends.

Despite our apparent success in the use of PSA as a screening tool, recent systematic analysis suggested that up to $60 \%$ of Adenocarcinomas of the Prostate can be safely observed without a need for immediate intervention [24].

Therefore, the next question has become at what age will early diagnosis prove to be most beneficial, and evidence suggests that the answer to this question lies in screening in the 55-69-year age range, as well as in patients at increased risk, because these patients will derive increased benefit from screening.

Since the USPSTF 2012 recommendation against PSA screening, there has been an overall decrease in PSA testing rates, varying between cohorts, age groups and physician specialty, with fewer younger men or those at higher risk and who might benefit, being screened (under-screening) [6].

Coincidently a reduction in the incidence of Adenocarcinoma of the Prostate has occurred even as we have seen an increase in the rates of advanced disease at the time of presentation, characterized by increasing pre-biopsy PSA levels, higher Gleason scores and more advanced TNM stages [6] [9] [51].

Advocates of PSA testing have attributed the reduction in mortality from Prostate Cancer over the last several decades to the delayed effect of early detection initiatives [5] [40]. Although there is evidence that PSA screening can lead to over treatment of low risk disease, creating unnecessary morbidity and cost, when national trends in mortality are contrasted against the incidence figures, relatively few men die of the disease [5] [12] [49] [52].

The 2012 USPSTF recommendation against screening for Adenocarcinoma of the Prostate was made solely due to the risks of biopsy and subsequent treatment, however, since individual patients may place different weight on the potential harm of screening when compared with the benefit of a possible cure it has become incumbent for the medical community to engage in shared decision-making [49]. Further, while the USPSTF decision stressed the morbidity associated with radical prostatectomy and radiotherapy, it overlooked the morbidity associated with advanced and fatal disease; and it is incumbent upon the 
medical community not to dismiss the need for diagnosis [6].

We must spend more of our time in deliberation with our patients over treatment options and continue efforts in bringing to our patients methods to pro-actively identify biologically significant cancers in order to reduce harm to those who might achieve little to no benefit from treatment of clinically insignificant disease.

Incorporation of the newer screening tests for Adenocarcinoma of the Prostate into our diagnostic algorithms may be helpful in achieving these goals, and their inclusion as part of the standard urologic armamentarium for biopsy decisions, risk stratification and treatment selection should become standard [23].

Continued refinement and validation of PSA, the use of fractionated PSA, PHI, 4K scoring and PCA3, among other studies, may lead to a reduction in the diagnosis and treatment of indolent, non-life-threatening Adenocarcinoma of the Prostate while at the same time improving the identification and therefore treatment of those patients who harbor high-risk, aggressive disease at an earlier age and stage.

Therefore, screening should target any symptomatic individual, men in their sixth decade of life, men with risk factors such as family history of Adenocarcinoma of the Prostate, known genetic predisposition or African-American ethnicity where there exist clear benefits in making an early diagnosis and embarking on proven therapies.

\section{References}

[1] Wein, A.J., Kavoussi, L.R., Partin, A.W. and Peters, C.A. (2016) Campbell-Walsh Urology. Eleventh Edition.

[2] McAninch, J.W. and Lue, T.F. (2013) Smith and Tanagho's General Urology. 18th Edition.

[3] Kumar, V., Abbas, A.K., Fausto, N. and Aster, J.C. (2010) Robbins and Cotran Pathologic Basis of Disease. Eighth Edition.

[4] National Cancer Institute (2016) Surveillance Epidemiology and End Results Program (SEER). Cancer Stat Facts: Prostate Cancer 1975-2014.

https://seer.cancer.gov/statfacts/html/prost/html

[5] Salinas, C.A., Tsodikov, A., Ishak-Howard, M. and Cooney, K.A. (2014) Prostate Cancer in Young Men: An Important Clinical Entity. National Review of Urology, 11, 317-323. https://doi.org/10.1038/nrurol.2014.91

[6] Fleshner, K., Carlsson, S.V. and Roobol, M.J. (2017) The Effect of the USPSTF PSA Screening Recommendation on Prostate Cancer Incidence Patterns in the USA. National Review of Urology, 14, 26-37. https://doi.org/10.1038/nrurol.2016.251

[7] Frendl, D., et al. (2016) Impact of Guidelines on Prostate Cancer Screening in a Population-Based Setting, 2000-2014: Preliminary Results from the First AUA Data Grant. Journal of Urology, 195, e543. https://doi.org/10.1016/j.juro.2016.02.131

[8] Rosenberg, M., Crawford, D., Newmark, J. and Steiner, M. (2016) Use of PSA Screening Guidelines among Primary Care Physicians (Abstract MP39-01). Journal of Urology, 195, e541. https://doi.org/10.1016/j.juro.2016.02.126

[9] Olsson, C., Anderson, A. and Kapoor, D. (2016) Initial Prostate Cancer Detection 
before and after United States Preventative Task Force Recommendation on Prostate Cancer Screening. Journal of Urology, 195, e542. https://doi.org/10.1016/j.juro.2016.02.129

[10] Hussein, S., Satturwar, S. and Van der Kwast, T. (2015) Young Age Prostate Cancer. Journal of Clinical Pathology, 68, 511-515. https://doi.org/10.1136/jclinpath-2015-202993

[11] Grubb, R.L., Pinsky, P.F., Greenlee, R.T., et al. (2008) Prostate Cancer Screening in the Prostate, Lung, Colorectal and Ovarian Cancer Screening Trial: Update on Findings from the Initial Four Rounds of Screening in a Randomized Trial. BJU International, 102, 1524. https://doi.org/10.1111/j.1464-410X.2008.08214.x

[12] Hoffman, R.M., Meisner, A.L.W., Arap, W., Barry, M., Shah, S.K., Zeliadt, S.B. and Wiggins, C.L. (2016) Trends in United States Prostate Cancer Incidence Rates and Stage, 1995-2012. Cancer Epidemiology Biomarkers Prevention, 25, 259-263. https://doi.org/10.1158/1055-9965.EPI-15-0723

[13] American Cancer Society (2018) Facts and Figures 2018. American Cancer Society, Atlanta. www.cancer.org

[14] Brawley, O.W. (2012) Trends in Prostate Cancer in the United States. Journal of the National Cancer Institute. Monographs, 2012, 152-156.

https://doi.org/10.1093/jncimonographs/lgs035

[15] Haas, G.P., Delongchamps, N., Brawley, O.W., Wang, C.Y. and de la Rosa, G. (2008) The Worldwide Epidemiology of Prostate Cancer: Perspectives from Autopsy Studies. Canadian Journal of Urology, 15, 3866-3871.

[16] McDavid, K., Lee, J., Fulton, J.P., Tonita, J. and Thompson, T.D. (2004) Prostate Cancer Incidence and Mortality Rates and Trends in the United States and Canada. Public Health Reports, 119, 174-186.

[17] Moyer, Virginia on Behalf of the USPSTF (2012) US Preventive Services Task Force Recommendation Statement: Screening for Prostate Cancer. Annals of Internal Medicine, 157, 120-134.

[18] Lim, L.S., Sherin, K. and the ACPM Prevention Practice Committee (2008) Screening for Prostate Cancer in U.S. Men: ACPM Position Statement on Preventive Practice. American Journal of Preventive Medicine, 34, 164-170.

[19] Murtaza, M., Salih, A.F., Illzam, E.M., Sharifa, A.M., et al. (2016) Prostate Cancer: Pathophysiology, Diagnosis, and Prognosis. IOSR Journal of Dental and Medical Sciences, 15, 122-126.

[20] Moore, K.L., Dalley, A.F. and Agur, A.M.R. (2010) Clinically Oriented Anatomy. Sixth Edition.

[21] Hess, K.R., Varadhachary, G.R., Taylor, S.H., et al. (2006) Metastatic Patterns in Adenocarcinoma. Cancer, 106, 1624-1633. https://doi.org/10.1002/cncr.21778

[22] Velcheti, V., Karnik, S., Bardot, S.F. and Prakash, O. (2008) Pathogenesis of Prostate Cancer: Lessons from Basic Research. The Ochsner Journal, 8, 213-218.

[23] Loeb, S. and Catalona, W.J. (2014) The Prostate Health Index: A New Test for the Detection of Prostate Cancer. Therapeutic Advances Urology, 6, 74-77. https://doi.org/10.1177/1756287213513488

[24] Punnen, S., Pavan, N. and Parekh, D.J. (2015) Finding the Wolf in Sheep's Clothing: the 4 Kscore Is a Novel Blood Test That Can Accurately Identify the Risk of Aggressive Prostate Cancer. Reviews in Urology, 17, 3-13.

[25] Vickers, A.J., et al. (2010) Prostate Specific Antigen Concentration at Age 60 and Death or Metastasis from Prostate Cancer: Case-Control Study. British Medical 
Journal, 341, c4521. https://doi.org/10.1136/bmj.c4521

[26] Marks, L.S., Fradet, Y., Deras, H., et al. (2007) PCA3 Molecular Urine Assay for Prostate Cancer in Men Undergoing Repeat Biopsy. Urology, 69, 532-535. https://doi.org/10.1016/j.urology.2006.12.014

[27] Deras, H., Aubin, S.M., Blasé, A., et al. (2008) PCA3: A Molecular Urine Assay for Predicting Prostate Biopsy Outcome. Journal Urology, 179, 1587-1592. https://doi.org/10.1016/j.juro.2007.11.038

[28] Stephan, C., Jung, K., Semjonow, A., Schulze-Forster, K., Cammann, H., Hu, X., et al. (2013) Comparative Assessment of Urinary Prostate Cancer Antigen 3 and TMPRSS2: ERG Gene Fusion with the Serum [-2] Pro-Prostate Specific Antigen Based Prostate Health Index for Detection of Prostate Cancer. Clinical Chemistry, 59, 280-288.

[29] Lin, D.W., Porter, M. and Montgomery, B. (2009) Treatment and Survival Outcomes in Young Men Diagnosed with Prostate Cancer: A Population Based Cohort Study. Cancer, 115, 2863-2871. https://doi.org/10.1002/cncr.24324

[30] Lange, E.M., Salinas, C.A., Zuhike, K.A., Ray, A.M., Wang, Y., et al. (2012) Early Onset Prostate Cancer Has a Significant Genetic Component. Prostate, 72, 147-156. https://doi.org/10.1002/cncr.24324

[31] Lange, E.M., Johnson, A.M., Wang, Y., et al. (2014) Genome-Wide Association Scan for Variants Associated with Early-Onset Prostate Cancer. PLoS ONE, 9, e93436. https://doi.org/10.1371/journal.pone.0093436

[32] Al-Ahmadie, H.A., Tickoo, S.K., Olgac, S., et al. (2008) Anterior-Predominant Prostatic Tumors: Zone of Origin and Pathologic Outcomes at Radical Prostatectomy. American Journal of Surgical Pathology, 32, 229-235. https://doi.org/10.1097/PAS.0b013e31812f7b27

[33] Tomlins, S.A., Bjartell, A., Chinnaiyan, A.M., et al. (2009) ETS Gene Fusions in Prostate Cancer: From Discovery to Daily Clinical Practice. European Urology, 56, 275-286. https://doi.org/10.1016/j.eururo.2009.04.036

[34] Young, A., Palanisamy, N., Siddiqui, J., et al. (2012) Correlation of Urine TMPRSS2: ERG and PCA3 to ERG+ and Total Prostate Cancer Burden. American Journal of Clinical Pathology, 138, 685-696. https://doi.org/10.1309/AJCPU7PPWUPYG8OH

[35] Narod, S.A., et al. (2008) Fusion in the ETS Gene Family and Prostate Cancer. British Journal of Cancer, 99, 847-851. https://doi.org/10.1038/sj.bjc.6604558

[36] Roobol, M.J., Kerkhof, M., Schroder, F.H., et al. (2009) Prostate Cancer Mortality Reduction by Prostate Specific Antigen Based Screening Adjusted for Non-Attendance and Contamination in the European Randomized Study of Screening for Prostate Cancer (ERSPC). European Urology, 56, 584.

https://doi.org/10.1016/j.eururo.2009.07.018

[37] Moore, C.M., Robertson, N.L., Arsanious, N., Middleton, T., et al. (2013) Image-Guided Prostate Biopsy using Magnetic Resonance Imaging-Derived Targets: A Systematic Review. European Urology, 63, 125. https://doi.org/10.1016/j.eururo.2012.06.004

[38] Siddiqui, M.M., Rais-Bahrami, S., Truong, H., Stamatakis, L., et al. (2013) Magnetic Resonance Imaging/Ultrasound-Fusion Biopsy Significantly Upgrades Prostate Cancer versus Systematic 12-Core Transrectal Ultrasound Biopsy. European Urology, 64, 713-719. https://doi.org/10.1016/j.eururo.2013.05.059

[39] Andriole, G.L., Crawford, D.E., Grub III, R.L., et al. (2012) Prostate Cancer Screening in the Randomized Prostate, Lung, Colorectal, and Ovarian Cancer Screening Trial: Mortality Results after 13 Years of Follow up. Journal of the National Cancer 
Institute, 104, 125-132. https://doi.org/10.1093/jnci/djr500

[40] Schroder, F.H., et al. (2009) Screening and Prostate Cancer Mortality in a Randomized European Study. New England Journal of Medicine, 360, 1320-1328. https://doi.org/10.1056/NEJMoa0810084

[41] Andriole, G.L., Crawford, E.D., Grubb, R.L., et al. (2009) Mortality Results from a Randomized Prostate-Cancer Screening Trial. New England Journal of Medicine, 360, 1310. https://doi.org/10.1056/NEJMoa0810696

[42] Schroder, F.H., Hugosson, J., Roobol, M.J., Tammela, T.L.J., et al. (2014) The European Randomized Study of Screening for Prostate Cancer-Prostate Cancer Mortality at 13 Years of Follow-Up. The Lancet, 384, 2027-2035.

https://doi.org/10.1016/S0140-6736(14)60525-0

[43] Ries, L.A.G., Melbert, D., Krapcho, M., et al. (2007) SEER Cancer Statistic Review, 1975-2004. National Cancer Institute, Bethesda.

http://seer.cancer.gov/csr/1975_2004/

[44] United States Preventive Services Task Force (2017) Draft Recommendation Statement Regarding Prostate Cancer Screening.

https://www.uspreventiveservicestaskforce.org/Page/Document/draft-recommendat ion-statement/prostate-cancer-screening1

[45] United States Preventive Services Task Force (2012) Prostate Cancer Screening.

[46] Cooperberg, M.R., et al. (2010) Time Trends and Local Variation in Primary Treatment of Localized Prostate Cancer. Journal of Clinical Oncology, 28, 1117. https://doi.org/10.1200/JCO.2009.26.0133

[47] Carroll, P.R., Parsons, J.K., Andriole, G., Bahnson, R.R., et al. (2017) NCCN Clinical Practice Guidelines in Oncology: Prostate Cancer Early Detection.

[48] Carter, B.H., Albertsen, P.C., Barry, M.J., Etzioni, R., et al. (2015) American Urological Association (AUA) Guideline. Early Detection of Prostate Cancer. http://www.auanet.org

[49] Talcott, J.A. (2015) In the Clinic: Prostate Cancer. Annals of Internal Medicine, ITC1-12. https://doi.org/10.7326/AITC201512010

[50] Jemal, A., et al. (2015) Prostate Cancer Incidence and PSA Testing Patterns in Relation to USPSTF Screening Recommendations. JAMA, 314, 2054-2061. https://doi.org/10.1001/jama.2015.14905

[51] Gaylis, F., et al. (2016) The Chance in Prostate Cancer Presentation and Diagnosis Coinciding with Screening Recommendations. Journal of Clinical Oncology, 34, 44.

[52] Boyle, P. and Brawley, O.W. (2009) Prostate Cancer: Current Evidence Weighs against Population Screening. Cancer Journal Clinics, 59, 220-224.

https://doi.org/10.3322/caac.20025 


\section{Abbreviations}

American Urologic Association (AUA),

Androgen Receptor (AR),

Atypical Small Acinar Proliferation (ASAP),

Benign Prostatic Hypertrophy (BPH),

Body Mass Index (BMI),

Breast Cancer Susceptibility Gene (BRCA),

Cancer of the Prostate Risk Assessment (CAPRA),

Digital Rectal Exam (DRE),

Dihydroepiandrostenedione (DHEA),

Dihydrotestosterone (DHT),

Early Detection (ED),

European Randomized Study of Screening for Adenocarcinoma of the Prostate (ERSPC),

Genome Wide Association Studies (GWAS),

Gleason Score, Kallikrein (KLK; hK),

Positive Predictive Value (PPV),

Prostate Cancer (PC),

Prostate Cancer Gene 3 (PCA3),

Prostate Cancer Prevention Trial (PCPT),

Prostate Health Index (PHI),

Prostate Intraepithelial Neoplasia (PIN),

Prostate Specific Antigen (PSA),

PSA Density (PSAD),

PSA Velocity (PSAV),

Prostate, Lung, Colorectal and Ovarian Cancer Screening Trial (PLCO),

Prostatic Acid Phosphatase (PAP),

Prostatic Adenocarcinoma (PA),

PSA Screening, Reduction by Dutasteride of Prostate Cancer Events (REDUCE),

Relative Risk (RR),

Risk Factors (RF),

Single Nucleotide Polymorphisms (SNP),

Transmembrane Protease, Serine (TMPRSS),

Transrectal Ultrasound (TRUS),

United States Preventive Services Task Force (USPSTF). 